University of Nebraska - Lincoln

DigitalCommons@University of Nebraska - Lincoln

John R. Hardy Papers

Research Papers in Physics and Astronomy

3-15-1970

Asymptotic Lattice Displacements about Point Defects in Cubic

Metals

John W. Flocken

University of California, Livermore, California

John R. Hardy

University of Nebraska - Lincoln

Follow this and additional works at: https://digitalcommons.unl.edu/physicshardy

Part of the Physics Commons

Flocken, John W. and Hardy, John R., "Asymptotic Lattice Displacements about Point Defects in Cubic Metals" (1970). John R. Hardy Papers. 24.

https://digitalcommons.unl.edu/physicshardy/24

This Article is brought to you for free and open access by the Research Papers in Physics and Astronomy at DigitalCommons@University of Nebraska - Lincoln. It has been accepted for inclusion in John R. Hardy Papers by an authorized administrator of DigitalCommons@University of Nebraska - Lincoln. 


\title{
Asymptotic Lattice Displacements about Point Defects in Cubic Metals*
}

\author{
John W. Flocken† and John R. Hardy \\ Lawrence Radiation Laboratory, University of California, Livermore, California 94550
}

(Received 5 May 1969)

\begin{abstract}
We have calculated the asymptotic displacements (a) about a single vacancy in $\mathrm{Al}, \mathrm{Na}, \mathrm{K}$, and $\mathrm{Li}$, (b) about a single interstitial $\mathrm{Cu}$ atom in a $\mathrm{Cu}$ host lattice, and (c) about a unit single double force along a $\langle 100\rangle$ direction in $\mathrm{Al}$ and $\mathrm{Cu}$. These calculations were made using the asymptotic equations of the method of lattice statics which, in its full form, is based on the Fourier transformation of the direct-space force equations between the detect and the host atoms in a large "supercell" of the lattice. Results were also obtained for each of the defect types in $\mathrm{Al}$ and $\mathrm{Cu}$ by means of an alternative approach proposed by Lie and Koehler. The asymptotic displacements around the spherical defects in $\mathrm{Al}, \mathrm{Cu}, \mathrm{Na}$, and $\mathrm{K}$ were compared with corresponding results obtained using the exact method of lattice statics. From this comparison it appears that elasticity theory cannot be justifiably applied closer than the $(4,4,4)$ neighbor to the defect in $\mathrm{Al}$, the $(5,4,4)$ neighbor in $\mathrm{Cu}$, and the 26th or the 27th neighbors in $\mathrm{Na}$ and $\mathrm{K}$. We also find large displacements along $\langle 110\rangle$ in $\mathrm{Al}$ and along $\langle 111\rangle$ in $\mathrm{Cu}, \mathrm{Na}$, and $\mathrm{K}$. The displacements obtained using a Green's-function technique developed by Lie and Koehler agree well with our results for the spherical detect in $\mathrm{Al}$ and for the unit single double-force defects in $\mathrm{Al}$ and $\mathrm{Cu}$. There are significant differences in the $\langle 100\rangle$ directions between the two sets of results for a spherical detect in $\mathrm{Cu}$. In general, the asymptotic method of lattice statics appears to be more exact and to involve fewer computational manipulations than the method of Lie and Koehler.
\end{abstract}

\section{INTRODUCTION}

W HEN prevailing techniques are used to calculate atomic displacements in the vicinity of a defect, the crystal is generally divided into two regions. In region I, near the defect, the host atoms are treated on a discrete basis, while in region II, the crystal is assumed to be an elastic continuum. However, it has recently been pointed out ${ }^{1-3}$ that when such semidiscrete methods are applied, the boundary between the two regions is generally assumed to be close to the defect. The resultant displacement field computed in this way is a very poor representation of the lattice distortion field far from the defect.

In 1957, Kanzaki ${ }^{4}$ presented a strictly atomistic method for calculating the displacements due to a defect. This method, which we refer to as the "method of lattice statics," is based on Fourier transforming the direct-space equilibrium equations. This transformation to reciprocal space decouples the $3 N \times 3 N$ equilibrium equations to $N 3 \times 3$ equations which may be solved explicitly for the reciprocal-space Fourier amplitudes. To define the wave vectors in the Fourier series one imposes periodic boundary conditions across the faces of a supercell having the same symmetry as the primitive lattice cell. This is equivalent to solving the problem of a lattice with one defect per supercell. This constraint leads in turn to the existence of only $N$ independent wave vectors, $\mathbf{q}$, contained within the first Brillouin zone. Each of the $3 \times 3$ equations then can be solved by specific

\footnotetext{
* Work performed in part under the auspices of the U. S. Atomic Energy Commission.

$\dagger$ Permanent address: Behlen Laboratory of Physics, University of Nebraska, Lincoln, Nebr.

$\ddagger J$. R. Hardy is a Professor of Physics at the University of Nebraska.

1 J. R. Hardy and R. Bullough, Phil. Mag. 17, 833 (1968).

2 J. R. Hardy, J. Phys. Chem. Solids 29, 2009 (1968).

3 J. W. Flocken and J. R. Hardy, Phys. Rev. 175, 919 (1968).

${ }^{4}$ H. Kanzaki, J. Phys. Chem. Solids 2, 24 (1957).
}

matrix inversion, and the Fourier amplitudes can be obtained by back-transforming the Fourier series.

Not only is the method of lattice statics inherently free from the shortcomings of the semidiscrete methods, but in the long-wavelength limit, the lattice-statics equations for the Fourier amplitudes of the displacement field are exactly analogous to the corresponding equations obtained from continuum elasticity. Since, as we shall show, the lattice displacements at large distances from the defect are entirely determined by these long-wave Fourier amplitudes, it is evident that in this limit, lattice statics and continuum elasticity become identical. Hence, in the method of lattice statics, elasticity theory can be used in an independent calculation to reveal the way in which the strain field displacements approach the elastic limit with increasing distance from the defect.

Our major concern in the present paper is with the computation of the displacements in the elastic limit. Within this limit, it is adequate to replace the exact Fourier amplitudes of the displacement field by their limiting expressions for small q. One can then convert the Fourier series for the displacement field into a Fourier integral and extend the limits of integration to infinity. For an isotropic material, the Fourier integral can be evaluated explicitly, 3,5 to yield the familiar result that the displacement field is everywhere radial and falls off as $1 / r^{2}$, where $r$ is the distance from the defect. Although, as will be shown, the displacement field for anistropic materials still falls off as $1 / r^{2}$ along any given crystallographic direction, one cannot evaluate the components of the displacement field analytically for any general direction. Thus, our concern in this paper is with the application of the asymptotic theory to anisotropic materials; in particular, those materials in which the anisotropy is so large that one cannot hope to use

5 J. D. Eshelby, Solid State Phys. 3, 79 (1956). 
a perturbation approach (see Seeger et al..$^{6}$ ). Therefore, we have developed the equations for the components of the asymptotic displacements in such a way that the integrals for any desired direction can be evaluated numerically on a computer. In this way we have calculated the asymptotic displacements about an interstitial $\mathrm{Cu}$ atom in a $\mathrm{Cu}$ lattice and about isolated vacancies in $\mathrm{Al}, \mathrm{Na}, \mathrm{K}$, and Li.

The resultant displacements along certain symmetry directions can be compared with those in previous papers. ${ }^{1,3,7}$ To make this comparison for the two alkali metals we have found it necessary to compute the exact displacements for neighbors more distant than those considered in our previous work ${ }^{7}$; while for the vacancy in $\mathrm{Al}$, we are able to make direct comparison with the results obtained in a previous paper. ${ }^{1}$

It is possible to compare directly the present asymptotic results with those of an alternative method by Lie and Koehler, ${ }^{8}$ although it should be stressed that our results are exact, whereas theirs are approximate. One of the purposes of this comparison is to assess the reliability of their approximation for defects in $\mathrm{Cu}$ and Al. As might be expected in view of its low anisotropy, the comparison in the case of $\mathrm{Al}$ is good, but the agreement between the two sets of results for $\mathrm{Cu}$ is considerably worse.

In Sec. II we give a more detailed development of the asymptotic lattice-statics formalism. In Sec. III we discuss the application of this method to point defects in the materials mentioned above and give the appropriate results. In Sec. IV we discuss the comparison of the asymptotic results with those obtained by lattice-statics theory and with the results of Lie and Koehler. Section $\mathrm{V}$ will be devoted to a final summary of our calculations and results.

\section{METHOD OF LATTICE STATICS IN ASYMPTOTIC LIMIT}

The derivation of the equations of lattice statics has been presented in detail elsewhere, ${ }^{3,4}$ and we will summarize the important results at this point. We consider a point defect to be introduced at the center of a "supercell" containing $N$ host atoms and take as our reference system a set of Cartesian coordinates along the $\langle 100\rangle$ directions of the crystal with the defect situated at the origin. If the defect exerts a force, $\mathbf{F}^{l}$, on its $l$ th neighboring atom, we define the reciprocal-space generalized force array $\mathrm{F}_{\alpha} \mathrm{q}$ as

$$
F_{\alpha}^{\mathrm{q}} \equiv \sum_{l} F_{\alpha}^{l} \exp \left(-i \mathrm{q} \cdot \mathbf{r}^{l}\right)
$$

where $\mathbf{r}^{l}$ is the position vector of the $l$ th atom, $\mathbf{q}$ is a wave vector in the first Brillouin zone (FBZ), and $\alpha$ refers to one of the coordinate axes and ranges from 1-3.

\footnotetext{
${ }^{6}$ A. Seeger, E. Mann, and R. von Jan, J. Phys. Chem. Solids 23, 639 (1962)

${ }^{7}$ J. W. Flocken and J. R. Hardy, Phys. Rev. 177, 1054 (1969).

8 K. N. C. Lie and J. S. Koehler, Advan. Phys. 17, 421 (1968).
}

Similarly, if $V_{\alpha \beta}{ }^{0 l}$ is the direct-space force-constant matrix, the Fourier-transformed force-constant matrix $V_{\alpha \beta}{ }^{-q}$ is defined by

$$
V_{\alpha \beta^{-q}}=\sum_{l} V_{\alpha \beta}{ }^{0 l} \exp \left(i \mathrm{q} \cdot \mathbf{r}^{l}\right) .
$$

Then the displacement $\xi^{l}$ of the $l$ th atom can be shown to be given by

$$
\xi^{l}=\frac{i}{N} \sum_{\mathrm{q}} \mathbf{Q}^{\mathrm{q}} \sin \left(\mathbf{q} \cdot \mathbf{r}^{l}\right)
$$

where the Fourier amplitudes $\mathbf{Q}^{q}$ are given by the matrix equation

$$
\mathbf{Q}^{\mathrm{q}}=\left(\mathrm{V}^{-\mathrm{q}}\right)^{-1} \mathbf{F}^{\mathrm{q}} .
$$

As $\mathbf{r}^{l}$ becomes large, the sine term in Eq. (3) oscillates rapidly so that the dominant contribution to the sum arises from the region of $q$ space about the origin. Therefor we can replace $\mathbf{Q}^{q}$ by its limiting value for small $\mathbf{q}$ and when we convert the sum in Eq. (3) to an integral, we have

$$
\xi^{l}=\frac{i v}{(2 \pi)^{3}} \iiint_{\text {FBZ }} \mathbf{Q}^{\mathbf{q} \rightarrow 0} \sin \left(\mathbf{q} \cdot \mathbf{r}^{l}\right) d^{3} \mathbf{q},
$$

where $v$ is the volume of the unit cell in direct space. (For a fcc cell of side $2 a, v=2 a^{3}$, and for a bcc lattice, $v=4 a^{3}$.)

For any cubic monatomic crystal, the generalized force array, expanded to first order in $\mathbf{q}$, can be put into the form

$$
F_{\alpha} \mathrm{q}=(-i G / a) k_{\alpha},
$$

where $\mathbf{k}=\mathrm{q} a . G$ is a constant which depends on the detailed structure of the defect. It can be evaluated according to the prescription given by $\mathrm{Hardy}^{2}$

$$
G_{\alpha \beta}=\sum_{l} F_{\alpha}^{l} r_{\beta}^{l},
$$

where $F_{\alpha}{ }^{l}$ is the $\alpha$ th component of the direct-space force exerted by the defect on the $l$ th atom. These forces are obtained as results of exact lattice-statics calculations. Although $G_{\alpha \beta}$ is, in general, a tensor, it is diagonal for a defect of cubic symmetry and the diagonal elements are all equal.

When the $\mathrm{V}^{\mathrm{q}}$ matrix is expanded as a power series in $\mathbf{q}$, the lowest nonvanishing terms are of second order in q. When these limiting forms of $\mathrm{V}^{\mathrm{q}}$ and $\mathbf{F}^{\mathrm{q}}$ are substituted into Eq. (4), the $\alpha$ th component of the directspace displacement for the $l$ th atom can be written as

$$
\begin{aligned}
& \xi_{\alpha}{ }^{l}=\frac{G}{(2 \pi)^{3} a^{2}} \\
& \times \iiint_{\mathrm{FBZ}} \frac{k_{\alpha}\left(E k^{4}+F k_{\alpha}{ }^{2} k^{2}+H k_{\beta}{ }^{2} k_{\gamma}{ }^{2}\right) \sin (\mathbf{k} \cdot \mathbf{L}) d d^{3 \mathbf{k}}}{P(k)},
\end{aligned}
$$


if $\alpha \neq \beta \neq \gamma \neq \alpha$; and where $\mathbf{k}=\mathrm{q} a, \mathbf{L}=\mathbf{r}^{l} / a$; and $\alpha$, and $\beta$, $\gamma$ refer to the Cartesian axes. $P(k)$ is the determinant of the inverse $\mathrm{V}^{\mathrm{q}}$ matrix and is given by

$$
\begin{aligned}
P(k)=D k^{6}+B k^{2}\left(k_{\alpha}{ }^{2} k_{\beta}{ }^{2}+k_{\beta}{ }^{2} k_{\gamma}{ }^{2}+\right. & \left.k_{\gamma}{ }^{2} k_{\alpha}{ }^{2}\right) \\
& +A\left(k_{\alpha}{ }^{2} k_{\beta}{ }^{2} k_{\gamma}{ }^{2}\right),
\end{aligned}
$$

again, with the restriction $\alpha \neq \beta \neq \gamma \neq \alpha$.

The constants which appear in Eq. (9) and under the integral in Eq. (8) can be expressed in terms of either the force constants or the elastic constants of the host lattice. In terms of the elastic constants, they are given by

$$
\begin{aligned}
A= & \left(C_{11}-C_{12}-2 C_{44}\right) \\
\quad & \quad \times\left[\left(C_{11}-C_{44}\right)\left(C_{11}+C_{12}\right)-2\left(C_{12}+C_{44}\right)^{2}\right], \\
B= & C_{44}\left(C_{11}+C_{12}\right)\left(C_{11}-C_{12}-2 C_{44}\right), \\
D= & C_{11} C_{44}, \\
E= & C_{44}\left(C_{11}-C_{12}-C_{44}\right), \\
F= & C_{44}\left(C_{12}+2 C_{44}-C_{11}\right), \\
H= & \left(C_{11}-C_{12}-2 C_{44}\right)^{2},
\end{aligned}
$$

where the $C_{i j}$ are the independent elastic constants for a cubic lattice. ${ }^{2}$

The integral in Eq. (8) is evaluated by the method due to Kanzaki. ${ }^{4}$ The Cartesian coordinates in reciprocal space are rotated about the origin so that the directspace position vector $\mathbf{r}^{l}$ lies along the $Z$ axis of the new set of coordinates, and the vector $\mathbf{k}$ is then expressed in polar coordinates in the new reference frame. Thus, in these coordinates,

$$
k_{\alpha}^{2}=k^{2} g_{\alpha}(\cos \theta, \phi),
$$

where $g_{\alpha}$ is a function of only the cosines of the polar angle $\theta .{ }^{9}$ Define $f_{\alpha}(\cos \theta, \phi)$ as

$$
f_{\alpha}(\cos \theta, \phi) \equiv \frac{E+F g_{\alpha}(\cos \theta, \phi)+H g_{\beta} g_{\gamma}}{D+B\left(g_{\alpha} g_{\beta}+g_{\beta} g_{\gamma}+g_{\gamma} g_{\alpha}\right)+A g_{\alpha} g_{\beta} g_{\gamma}},
$$

where the subscripts, $\alpha, \beta$, and $\gamma$ refer to the original Cartesian axes. Thus, Eq. (8) becomes

$$
\begin{aligned}
\xi_{\alpha}{ }^{l}=\frac{G}{(2 \pi)^{3} a^{2}} \iint_{\mathrm{FBZ}} \int_{\alpha} k_{\alpha} f_{\alpha} \\
\\
\quad \times(\cos \theta, \phi) \sin (\mathbf{k} \cdot \mathbf{L}) d k d(\cos \theta,) d \phi,
\end{aligned}
$$

${ }^{9}$ One must realize that the $g_{\alpha}(\cos \theta, \phi)$ are not unique in that their exact form depends on the transformation matrix used in aligning $\mathbf{r}^{l}$ with the $z$ axis. In the present case, we rotated the original coordinate system about the $z$ axis until $\mathbf{r}^{l}$ was in the $x z$ plane, then rotated about the "new" $y$ axis until $\mathrm{r}^{l}$ lay along $z$. The point is that however one accomplishes the transformation, he will arrive at a set of equations identical to those in Eq. (11), where $g_{\alpha}(\cos \theta, \phi)$ is some function, the only variables of which are $\cos \theta$ and $\phi$. The final asymptotic results must, of course, be independent of the transformation matrix and the exact form of the $g_{\alpha}(\cos \theta, \phi)$. which can be written

$$
\begin{aligned}
\xi_{\alpha}{ }^{l}=\frac{-G}{(2 \pi)^{3} a^{2}} & \frac{\partial}{\partial L_{\alpha}} \iint_{\mathrm{FBZ}} \int_{\alpha} f_{\alpha} \\
& \times(\cos \theta, \phi) \cos (k L \cos \theta) d k d(\cos \theta) d \phi .
\end{aligned}
$$

We now argue that, since the major contribution to the integral will come from points near the origin, we may extend the $k$ integral to infinity and introduce a convergence factor of the form $e^{-\epsilon|\mathrm{k}|}, \epsilon \rightarrow 0$, into the integral of Eq. (14). The $k$ integration then produces a $\delta$ function with argument of $\cos \theta$. Thus,

$$
\begin{aligned}
\xi_{\alpha}^{l}=\frac{-G}{2 \pi^{2} a^{2}} \frac{\partial}{\partial L_{\alpha}} \int_{-1}^{1} \int_{0}^{2 \pi} \frac{f_{\alpha}(\cos \theta, \phi)}{|\mathbf{L}|} \\
\times \delta(\cos \theta) d(\cos \theta) d \phi .
\end{aligned}
$$

Hence, integrating over $\cos \theta$, we obtain

$$
\xi_{\alpha}^{l}=\frac{-G}{2 \pi^{2} a^{2}} \frac{\partial}{\partial L_{\alpha}} \int_{0}^{2 \pi} \frac{f_{\alpha}(0, \phi)}{|\mathrm{L}|} d \phi .
$$

The remaining $\phi$ integral cannot, in general, be evaluated analytically for an anisotropic crystal. Since the parameter $\phi$ is not dependent on the direction of $\mathrm{L}$, we may take the derivative inside the integral and differentiate the integrand. After a good deal of algebraic manipulation, one arrives at an analytic form for the integrand and the integral has then to be evaluated numerically.

The generalized force array for a double force along the $\alpha$ direction is obtained by the same prescription as that used for the cubic defect; but Eq. (6) then has the form

$$
\mathrm{F}^{\mathrm{q}}=\frac{-i G^{\prime}}{a}\left(\begin{array}{c}
k_{\alpha} \\
0 \\
0
\end{array}\right),
$$

where $G^{\prime}$ is obtained from Eq. (7) exactly as for $G$. After substituting the asymptotic forms for the double-force generalized force array $\mathbf{F}^{q}$ and the force-constant matrix $\mathrm{V}^{-\mathrm{q}}$ into Eq. (3), we arrive at the following equations for the three components of displacement along the Cartesian coordinate axes:

$$
\begin{array}{r}
\xi_{\alpha}^{l}=\frac{G^{\prime}}{(2 \pi)^{3} a^{2}} \iint_{\mathrm{FBZ}} \int_{k_{\alpha}} \frac{E^{\prime} k^{4}+F^{\prime} k_{\alpha}{ }^{2} k^{2}+H^{\prime} k_{\beta}{ }^{2} k_{\gamma}{ }^{2}}{P(k)} \\
\times \sin (\mathbf{k} \cdot \mathbf{L}) d^{3} \mathbf{k},
\end{array}
$$

where $\alpha$ is along the [100] axis, parallel to the double force,

$$
\begin{array}{r}
\xi_{\beta} l=\frac{G^{\prime}}{(2 \pi)^{3} a^{2}} \iint_{\mathrm{FBZ}} \int_{\mathrm{B}} k_{\beta}\left(M k^{2}{k_{\alpha}}^{2}+N k_{\alpha^{2}} k_{\gamma}^{2}\right) \\
\times \sin (\mathbf{k} \cdot \mathbf{L}) d^{3} \mathbf{k},
\end{array}
$$




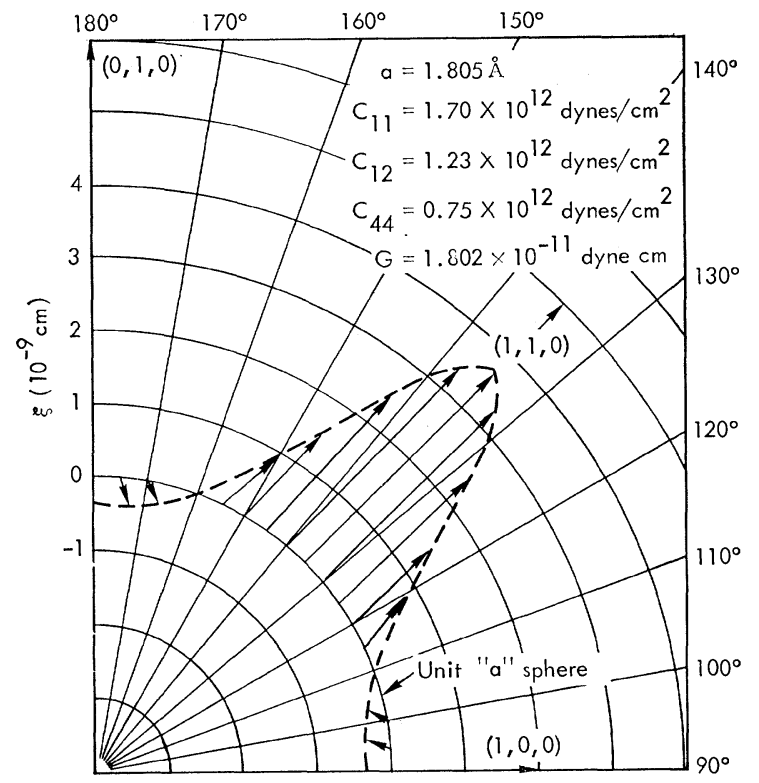

(a)

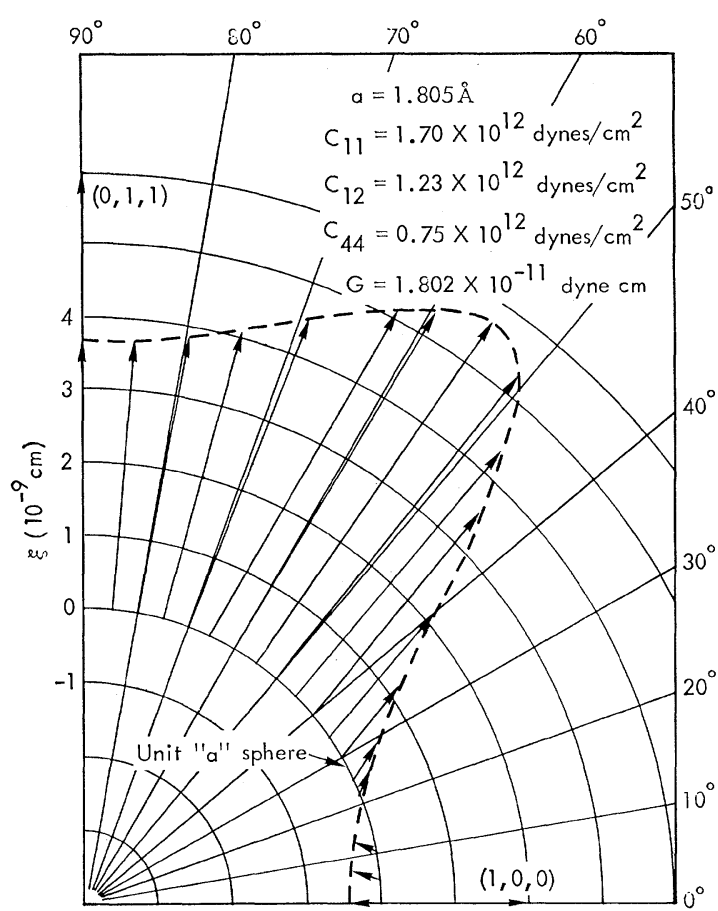

(b)

FIG. 1. Displacement profile in the first quadrant of the (001) plane about an interstitial $\mathrm{Cu}$ atom in $\mathrm{Cu}$; (b) displacement profile in the first quadrant of the (011) plane about an interstitial $\mathrm{Cu}$ atom in $\mathrm{Cu}$.

and the equation for $\xi_{\gamma}{ }^{l}$ is the same as Eq. (19), with the indices $\beta$ and $\gamma$ interchanged, and $\beta$ and $\gamma$ are perpendicular to the $[100]$ axis.

The constants in the numerator of the integrand are given by

$$
\begin{aligned}
E^{\prime} & =C_{11} C_{44}, \\
F^{\prime} & =C_{44}\left(C_{44}-C_{11}\right), \\
H^{\prime} & =C_{11}{ }^{2}-C_{12}{ }^{2}-2 C_{44}\left(C_{11}+C_{12}\right), \\
M & =-C_{44}\left(C_{12}+C_{44}\right), \\
N & =\left(C_{12}+C_{44}\right)\left(C_{12}-C_{11}+2 C_{44}\right) .
\end{aligned}
$$

TABLE I. Comparison of asymptotic displacements about an interstitial $\mathrm{Cu}$ atom in $\mathrm{Cu}$; (001) plane.

\begin{tabular}{ccccc}
\hline \hline \multicolumn{5}{c}{ Results } \\
$\begin{array}{c}c \\
\text { swept in } \\
\text { (011) } \\
\text { plane } \\
\text { (deg) }\end{array}$ & $\begin{array}{c}\text { Asymptotic lattice- } \\
\text { statics method } \\
\text { ment } \\
\left(10^{-9} \mathrm{~cm}\right)\end{array}$ & $\begin{array}{c}\theta \\
\text { (deviation } \\
\text { from } \\
\text { radial) }\end{array}$ & $\begin{array}{c}\text { Lie-Koehler method } \\
\text { ment } \\
\left(10^{-9} \mathrm{~cm}\right)\end{array}$ & $\begin{array}{c}\theta \\
\text { (deviation } \\
\text { from } \\
\text { radial) }\end{array}$ \\
\hline 0.0 & -0.353 & $180^{\circ} 0^{\prime}$ & -0.685 & $180^{\circ} 0^{\prime}$ \\
10.0 & -0.275 & $139^{\circ} \circ 6^{\prime}$ & -0.452 & $173^{\circ} 44^{\prime}$ \\
20.0 & 0.474 & $45^{\circ} 57^{\prime}$ & 0.482 & $29^{\circ} 26^{\prime}$ \\
30.0 & 1.629 & $15^{\circ} 16^{\prime}$ & 1.921 & $11^{\circ} 4^{\prime}$ \\
40.0 & 3.342 & $3^{\circ} 52^{\prime}$ & 3.121 & $3^{\circ} 13^{\prime}$ \\
45.0 & 3.698 & $0^{\circ} 0^{\prime}$ & 3.301 & $0^{\circ} 0^{\prime}$ \\
& & $(011) \mathrm{plane}^{\prime}$ & & \\
0.0 & -0.353 & $180^{\circ} 0^{\prime}$ & -0.685 & $180^{\circ} 0^{\prime}$ \\
30.0 & 1.658 & $20^{\circ} 32^{\prime}$ & 2.251 & $10^{\circ} 41^{\prime}$ \\
45.0 & 4.741 & $4^{\circ} 49^{\prime}$ & 4.635 & $3^{\circ} 35^{\prime}$ \\
60.0 & 5.486 & $-1^{\circ} 3^{\prime}$ & 5.023 & $-1^{\circ} 7^{\prime}$ \\
90.0 & 3.698 & $0^{\circ} 0^{\prime}$ & 2.800 & $0^{\circ} 0^{\prime}$ \\
\hline \hline
\end{tabular}

These integrals are evaluated in the same way as in Eq. (8). At present there are no existing calculations for double-force defects in cubic materials. We choose to leave the strength parameter $G^{\prime}$ as an arbitrary constant; and we have, therefore, made our calculations assuming a unit double force. As the strength parameter $G^{\prime}$ is simply a multiplicative factor in Eqs. (18) and (19) we can obtain the displacement field for any specific defect of this class by appropriately scaling the displacements produced by the unit double force.

TABLE II. Comparison of asymptotic displacements about a vacancy in $\mathrm{Al}$; (001) plane.

\begin{tabular}{ccc}
\hline \hline & $\begin{array}{c}\text { Asymptotic } \\
\text { lattice } \\
\text { statics } \\
\begin{array}{c}\epsilon \text { is } \\
\text { Displacements } \\
\left(10^{-9} \mathrm{~cm}\right)\end{array}\end{array}$ & $\begin{array}{c}\text { Displacement } \\
\text { from } \\
\text { Lie-Koehler } \\
\text { method } \\
\left(10^{-9} \mathrm{~cm}\right)\end{array}$ \\
\hline $0^{\circ} 0^{\prime}$ & -0.850 & -0.836 \\
$10^{\circ} 0^{\prime}$ & -0.888 & -0.886 \\
$20^{\circ} 0^{\prime}$ & -0.994 & -1.013 \\
$30^{\circ} 0^{\prime}$ & -1.129 & -1.156 \\
$40^{\circ} 0^{\prime}$ & -1.227 & -1.249 \\
$45^{\circ} 0^{\prime}$ & -1.242 & -1.262 \\
& $(0 \overline{1} 1)$ plane & \\
$0^{\circ} 0^{\prime}$ & -0.850 & -0.836 \\
$30^{\circ} 0^{\prime}$ & -1.149 & -1.177 \\
$45^{\circ} 0^{\prime}$ & -1.345 & -1.358 \\
$60^{\circ} 0^{\prime}$ & -1.376 & -1.383 \\
$90^{\circ} 0^{\prime}$ & -1.242 & -1.219 \\
\hline \hline
\end{tabular}




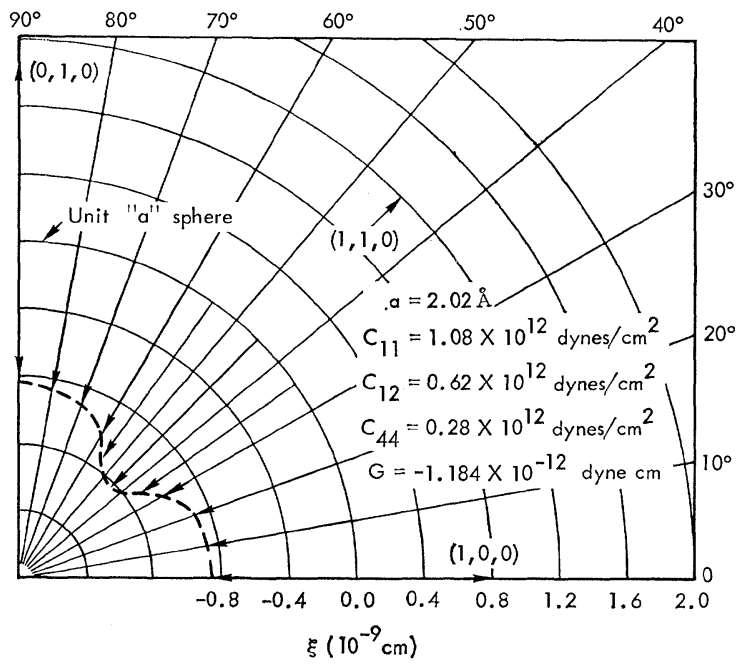

(a)

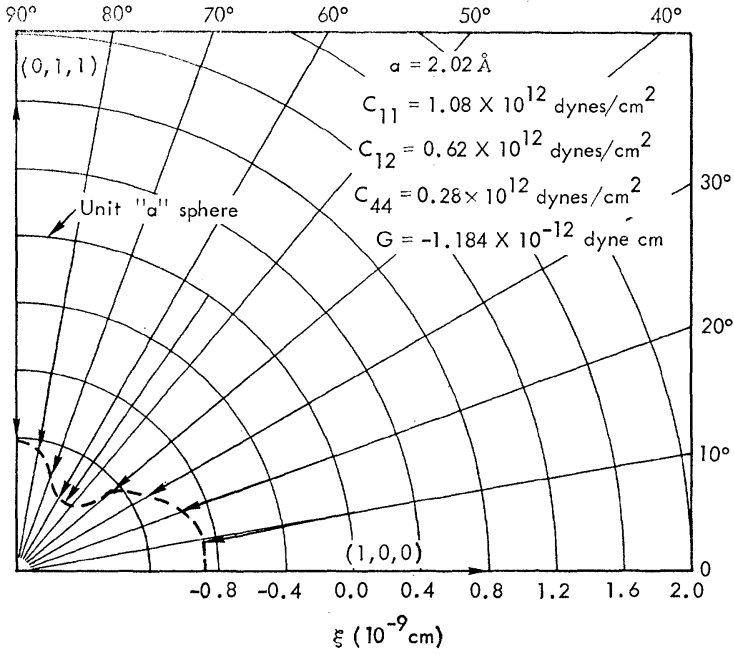

(b)

FIG. 2. (a) Displacement profile in the first quadrant of the (001) plane about a vacancy in $\mathrm{Al}$; (b) displacement profile in the first quadrant of the (011) plane about a vacancy in $\mathrm{Al}$.

\section{APPLICATIONS}

We have calculated the asymptotic displacements due to an interstitial $\mathrm{Cu}$ atom in $\mathrm{Cu}$ and due to isolated vacancies in $\mathrm{Al}, \mathrm{Na}, \mathrm{K}$, and $\mathrm{Li}$. We have also computed the displacements about a single double-force in $\mathrm{Cu}$ and Al. For comparison, we have used the approximate prescription given by Lie and Koehler ${ }^{8}$ for the calculation of elastic displacements due to unit triple double-forces (spherical defects) and unit single double-forces in $\mathrm{Cu}$ and $\mathrm{Al}$.

Since the displacements in a given direction fall off inversely as the square of the distance from the defect, we have chosen to represent our results as displacements of points originally on a sphere of radius " $a$ " from the defect. However, it is a simple matter to compute the displacement field of the crystal on any sphere of arbitrary radius $r$, since one simply scales by $(a / r)^{2}$.

The displacement profile has been computed at 2.5deg intervals in the $(0,0,1)$ and $(0, \overline{1}, 1)$ planes, and only the first quadrant of each profile is shown, since the entire profile can be generated by mirror reflection of this quadrant in the $x$ and $y$ axes.

The calculations were done on a CDC 3600. The numerical integrations were evaluated using Simpson's rule with 100 increments in $\phi$.

In Figs. 1(a) and 1(b) we show the appropriate displacement profiles for an interstitial $\mathrm{Cu}$ atom in $\mathrm{Cu}$. In Table I we compare the displacements calculated from the series of Lie and Koehler ${ }^{8}$ with the asymptotic

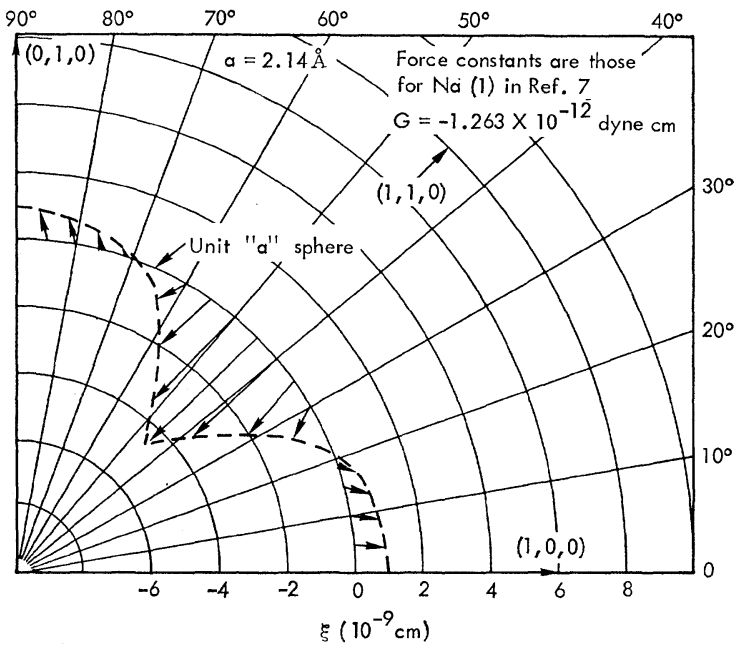

(a)

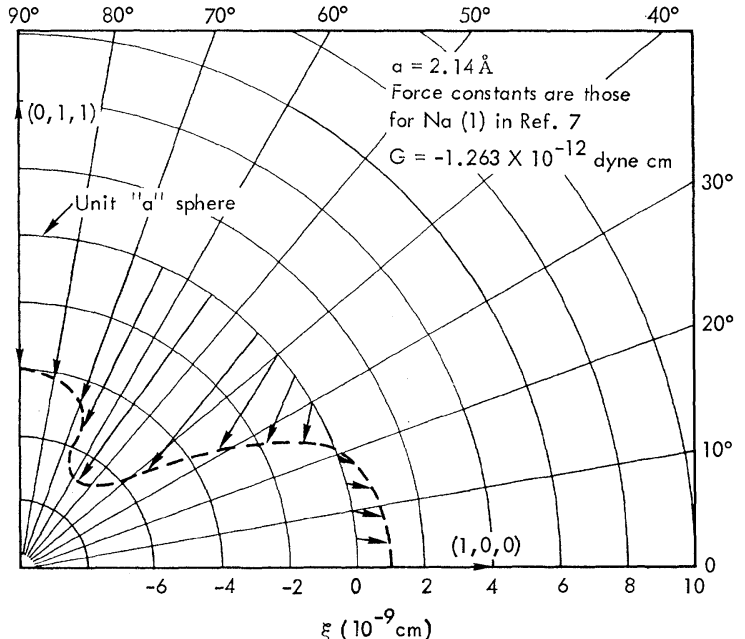

(b)

FIG. 3. (a) Displacement profile in the first quadrant of the (001) plane about a vacancy in $\mathrm{Na}$; (b) displacement profile in the first quadrant of the (011) plane about a vacancy in Na. 


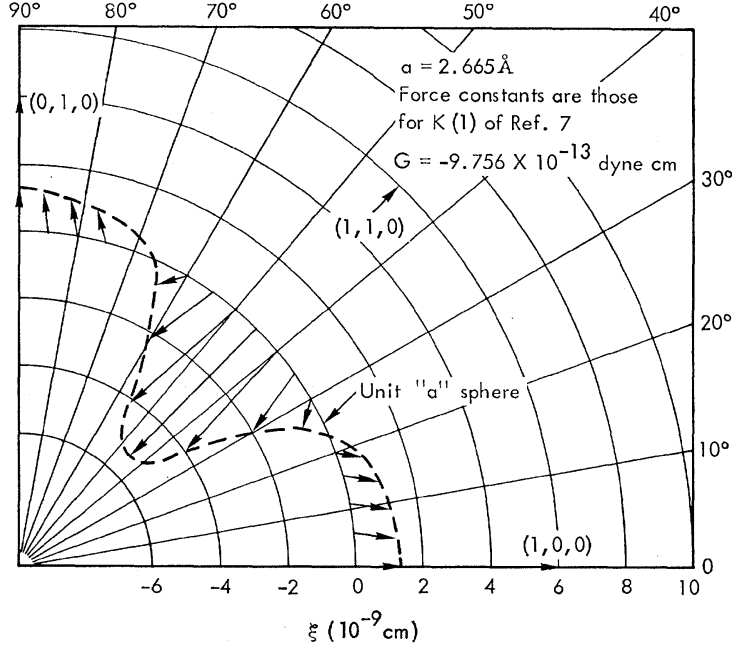

(a)

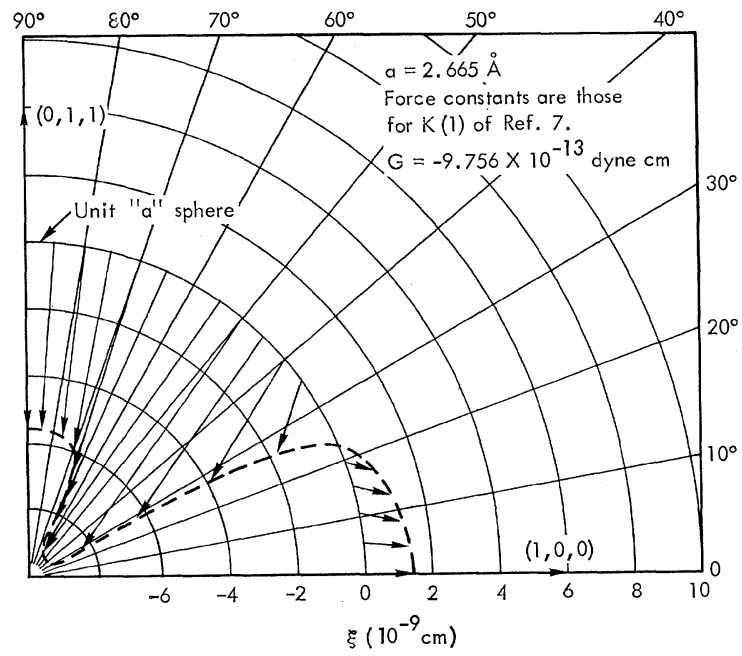

(b)

FIG. 4. (a) Displacement profile in the first quadrant of the (001) plane about a vacancy in K; (b) displacement profile in the first quadrant of the (011) plane about a vacancy in $K$.

lattice-statics values. Inward displacements are denoted by minus signs; $\theta$ is measured from the outward drawn radial. A negative value of $\theta$ indicates a clockwise deviation from the radial, and a positive value of $\theta$ indicates a counterclockwise deviation. Displacements for which $|\theta|$ is greater than $90^{\circ}$ are always inward.

In Figs. 2(a) and 2(b) we show the corresponding displacement profiles for Al. In Table II, we give a comparison of our results to those obtained by Lie and Koehler. In both cases the displacements are so nearly radial that we make no comparison of the predicted orientations. Figures 3(a), 3(b), 4(a), and 4(b) show the displacement profiles for vacancies in $\mathrm{Na}$ and $\mathrm{K}$. [The strengths are for the lattice models $\mathrm{Na}(1)$ and $\mathrm{K}(\mathbf{1})$ of Ref. 7.]

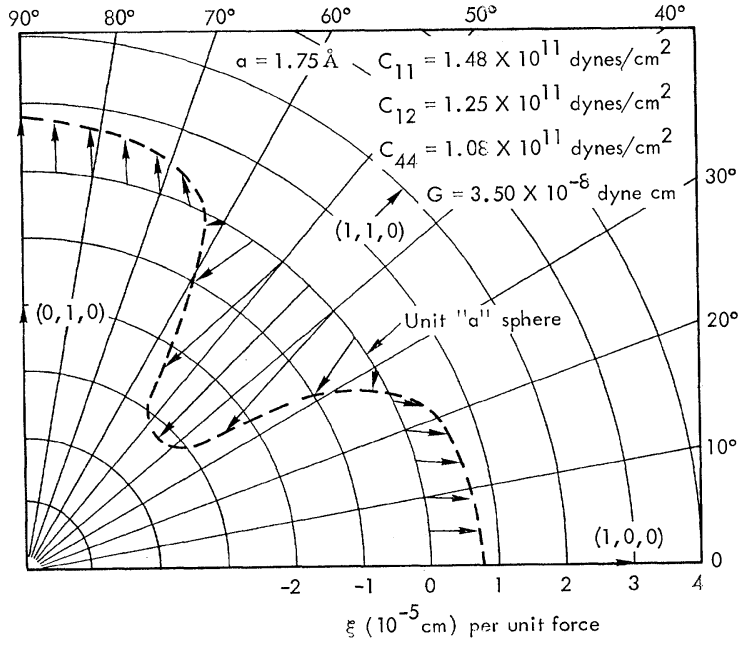

(a)
In Figs. 5(a) and 5(b) we show the displacement profiles for a vacancy in Li. In the case of this material, we have no knowledge of the strength parameter and we have therefore assumed a unit inward force on the nearest neighbors of the vacancy. It is evidently a simple matter to scale these displacements to those appropriate to the true elastic strength of the Li vacancy when this strength becomes known.

In Figs. 6(a), 6(b), 7(a), and 7(b), we show the displacement profiles for unit double forces along the [100] direction in $\mathrm{Cu}$ and $\mathrm{Al}$. In Tables III and IV we compare our exactly computed displacements with those computed by the Fourier-series approximation of Lie and Koehler.

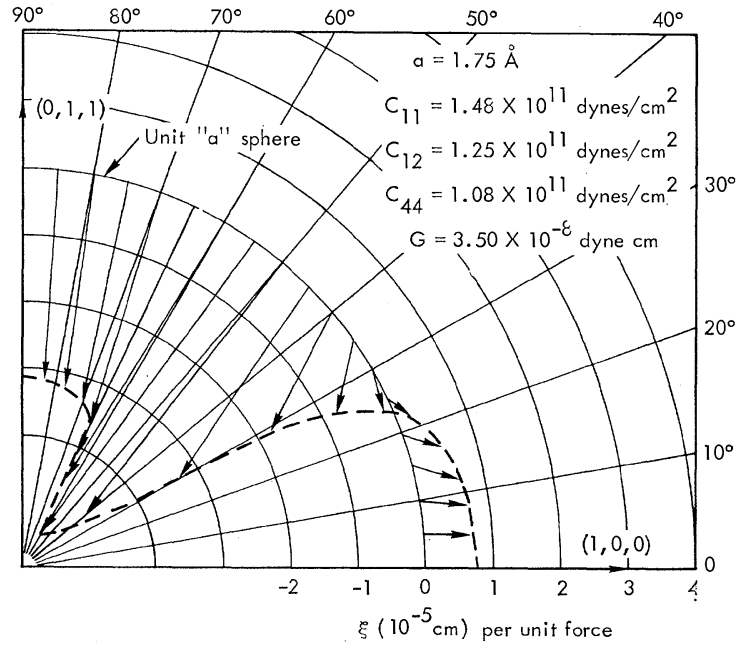

(b)
Frg. 5. (a) Displacement profile in the first quadrant of the (001) plane about a vacancy in Li; (b) displacement profile in the first quadrant of the (011) plane about a vacancy in Li. 


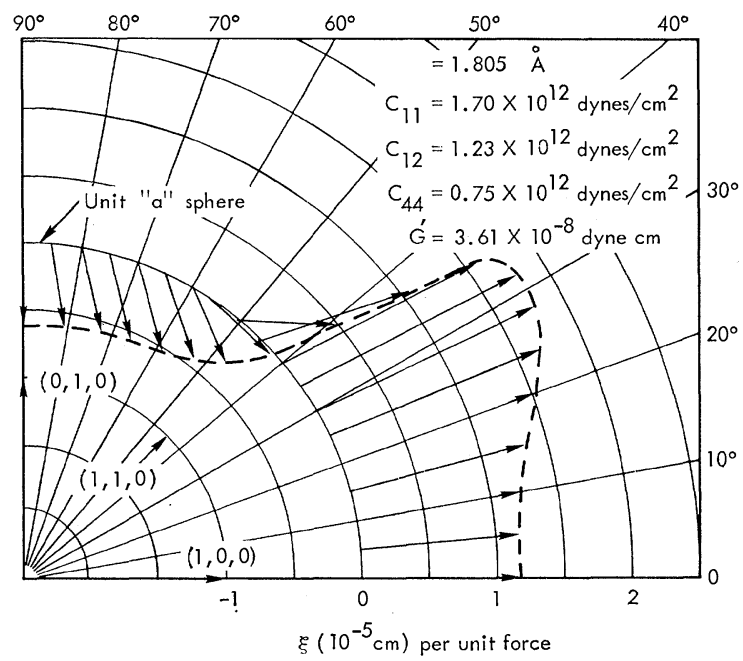

(a)

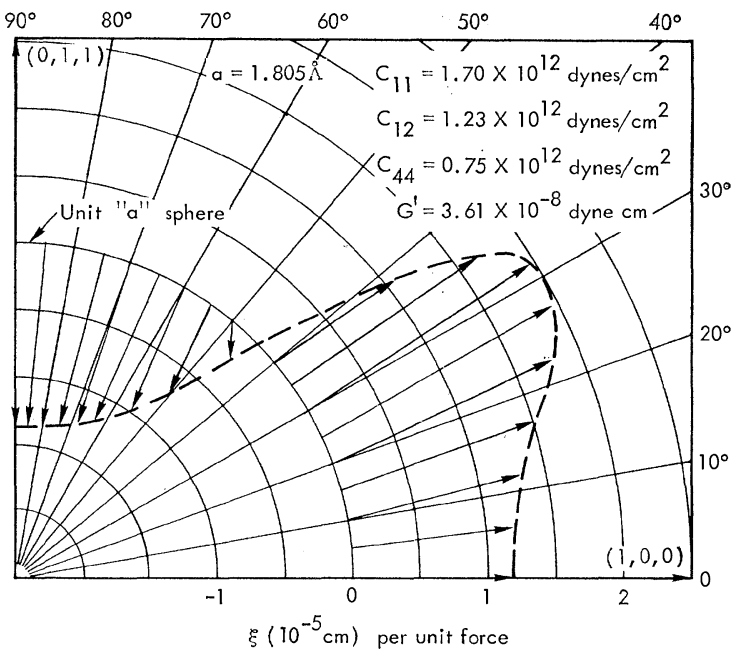

(b)

Frg. 6. (a) Displacement profile in the first quadrant of the (001) plane about a unit single double-force along $[100]$ in $\mathrm{Cu}$; (b) displacement profile in the first quadrant of the (011) plane about a unit single double-force along [100] in $\mathrm{Cu}$.

\section{DISCUSSION}

The strain field displacements calculated by exact lattice-statics may be compared to equivalent results from the asymptotic theory to get an indication of the distance from the defect at which elasticity theory may be justifiably applied. Values of $|\xi| r^{2}$ calculated by both the exact and asymptotic methods for lattice points about a vacancy in $\mathrm{Al}$ are given in Table V. A similar comparison is made in Table VI for lattice points about an interstitial $\mathrm{Cu}$ atom in $\mathrm{Cu}$. There are not enough points in most directions to demonstrate the approach of the displacements obtained from the exact theory to the elastic limit. Along $\langle 110\rangle$ in $\mathrm{Al}$ we find that the values of $|\xi| r^{2}$ remain well above the asymptotic value although along the $\langle 111\rangle$ directions, there is much closer agreement between the asymptotic values of $|\xi| r^{2}$ and those computed by lattice statics. However, in view of subsequent calculations it is apparent to us that it would be unwise to compare the asymptotic results to those given earlier by Hardy and Bullough ${ }^{1}$ since these are restricted to neighbors which are probably too close to the defect for the asymptotic theory to hold. Also, we believe there are certain problems associated with the use of a random sample of wave vectors for computing the displacements of very distant neighbors.

In the case of the interstitial $\mathrm{Cu}$ atom in $\mathrm{Cu}$, which we have treated earlier, ${ }^{3}$ we show in Table VI a comparison of the exact and asymptotic displacements. The former were calculated using a sample of 64000 regu-

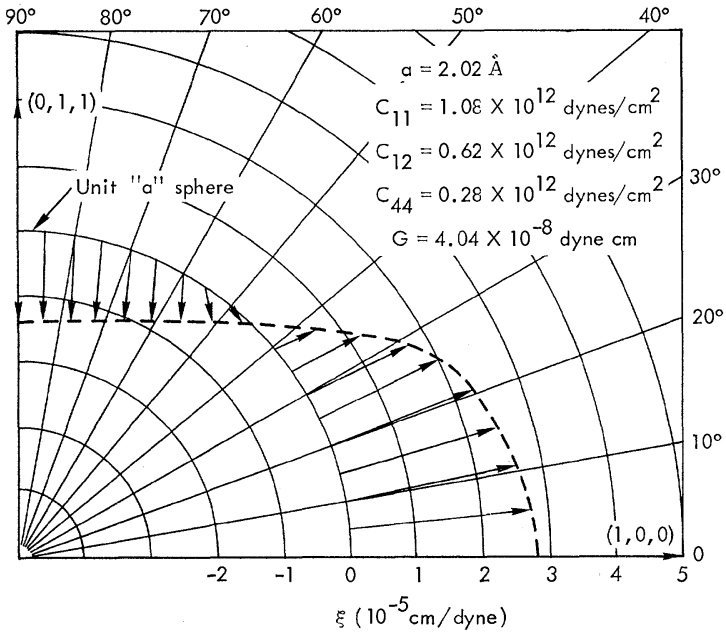

(a)

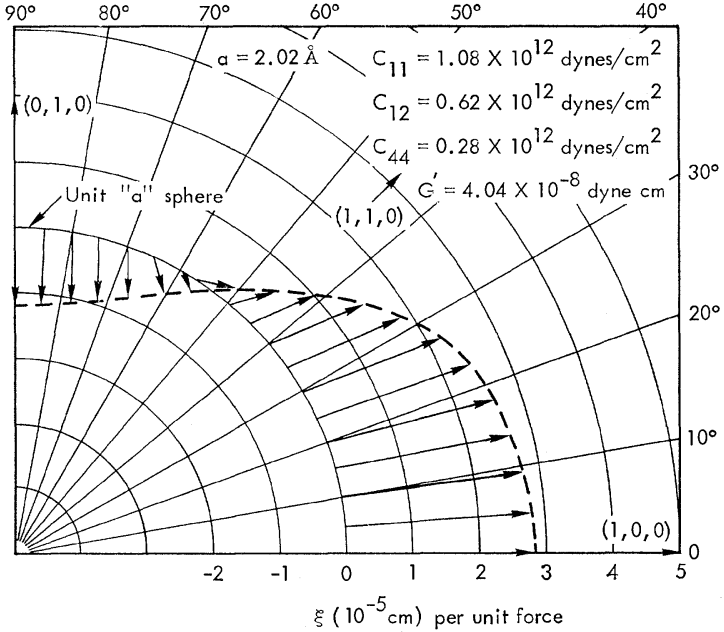

(b)

FIG. 7. (a) Displacement profile in the first quadrant of the (001) plane about a unit single double-force along [100] in $\mathrm{Al}$; (b) displacement profile in the first quadrant of the (011) plane about a unit single double-force along [100] in $\mathrm{Al}$. 
TABLE III. Comparison of asymptotic displacements about a unit single double-force along $[100]$ in $\mathrm{Cu} ;(001)$ plane.

\begin{tabular}{|c|c|c|c|c|}
\hline \multirow[b]{2}{*}{$\begin{array}{c}\boldsymbol{\epsilon} \\
\text { swept in } \\
(010) \\
\text { plane } \\
\text { (deg) } \\
\end{array}$} & \multicolumn{4}{|c|}{ Results } \\
\hline & $\begin{array}{c}\text { Asymptotic } \\
\text { Displace- } \\
\text { ments } \\
\left(10^{-5} \mathrm{~cm}\right)\end{array}$ & $\begin{array}{l}\text { lattice statics } \\
\theta \text { (deviation } \\
\text { from } \\
\text { radial) }\end{array}$ & $\begin{array}{c}\text { Lie-Koeh } \\
\text { Displace- } \\
\text { ments } \\
\left(10^{-5} \mathrm{~cm}\right)\end{array}$ & $\begin{array}{l}\theta \text { (deviation } \\
\text { from } \\
\text { radial) }\end{array}$ \\
\hline 0 & 1.176 & $0^{\circ} 0^{\prime}$ & 1.127 & $0^{\circ} 0^{\prime}$ \\
\hline 10 & 1.260 & $0^{\circ} 19^{\prime}$ & 1.264 & $0^{\circ} 36^{\prime}$ \\
\hline 20 & 1.511 & $-0^{\circ} 4^{\prime}$ & 1.580 & $-0^{\circ} 53^{\prime}$ \\
\hline 30 & 1.817 & $-4^{\circ} 31^{\prime}$ & 1.776 & $-4^{\circ} 55^{\prime}$ \\
\hline 40 & 1.614 & $-13^{\circ} 37^{\prime}$ & 1.497 & $-13^{\circ} 37^{\prime}$ \\
\hline 50 & 0.676 & $-53^{\circ} 30^{\prime}$ & 0.788 & $-41^{\circ} 43^{\prime}$ \\
\hline 60 & -0.635 & $-126^{\circ} 37^{\prime}$ & -0.648 & $-121^{\circ} 29^{\prime}$ \\
\hline 70 & -0.696 & $-144^{\circ} 0^{\prime}$ & -0.858 & $-150^{\circ} 37^{\prime}$ \\
\hline 80 & -0.652 & $-158^{\circ} 35^{\prime}$ & -0.652 & $-160^{\circ} 45^{\prime}$ \\
\hline 90 & -0.623 & $-180^{\circ} 0^{\prime}$ & -0.463 & $-180^{\circ} 0^{\prime}$ \\
\hline & & (01̄1) plane) & & \\
\hline 0 & 1.176 & $0^{\circ} 0^{\prime}$ & 1.127 & $0^{\circ} 0^{\prime}$ \\
\hline 10 & 1.343 & $4^{\circ} 22^{\prime}$ & 1.347 & $4^{\circ} 5^{\prime}$ \\
\hline 20 & 1.786 & $5^{\circ} 41^{\prime}$ & 1.870 & $5^{\circ} 33^{\prime}$ \\
\hline 30 & 2.000 & $3^{\circ} 2^{\prime}$ & 1.931 & $2^{\circ} 31^{\prime}$ \\
\hline 40 & 1.128 & $-5^{\circ} 7^{\prime}$ & 1.056 & $-5^{\circ} 5^{\prime}$ \\
\hline 50 & -0.293 & $-145^{\circ} 22^{\prime}$ & -0.264 & $-120^{\circ} 0^{\prime}$ \\
\hline 60 & -1.030 & $-174^{\circ} 50^{\prime}$ & -0.987 & $-173^{\circ} 24^{\prime}$ \\
\hline 70 & -1.288 & $-178^{\circ} 56^{\prime}$ & -1.335 & $-178^{\circ} 37^{\prime}$ \\
\hline 80 & -1.340 & $-180^{\circ} 2^{\prime}$ & -1.360 & $-179^{\circ} 30^{\prime}$ \\
\hline 90 & -1.343 & $-180^{\circ} 0^{\prime}$ & -1.335 & $-180^{\circ} 0^{\prime}$ \\
\hline
\end{tabular}

larly spaced wave vectors within the FBZ. Again, we feel that, except for some of the most distant neighbors, the asymptotic regime has not been reached. This in itself is a significant result, since it emphasizes once again the lack of validity of the continuum solution at what had previously been regarded as relatively large distances from the defect. In the case of the alkali metals

TABLE IV. Comparison of asymptotic displacements about a unit single double-force in $\mathrm{Al}$ along [100]; (001) plane.

\begin{tabular}{|c|c|c|c|c|}
\hline \multirow[b]{2}{*}{$\begin{array}{c}\epsilon \\
\text { swept in } \\
(001) \\
\text { plane } \\
\text { (deg) }\end{array}$} & \multicolumn{4}{|c|}{ Results } \\
\hline & \multicolumn{2}{|c|}{$\begin{array}{l}\text { Asymptotic lattice statics } \\
\text { Displace- } \theta \text { (deviation } \\
\text { ment } \\
\left(10^{-5} \mathrm{~cm}\right) \quad \text { from } \\
\text { radial) }\end{array}$} & \multicolumn{2}{|c|}{$\begin{array}{c}\text { Lie-Koihler method } \\
\text { Displace- } \theta \text { (deviation } \\
\text { ment } \\
\left(10^{-5} \mathrm{~cm}\right) \quad \text { from } \\
\text { radial) }\end{array}$} \\
\hline 0 & 2.814 & $0^{\circ} 0^{\prime}$ & 2.910 & $0^{\circ} 0^{\prime}$ \\
\hline 10 & 2.766 & $-1^{\circ} 50^{\prime}$ & 2.815 & $-2^{\circ} 15^{\prime}$ \\
\hline 20 & 2.585 & $-4^{\circ} 22^{\prime}$ & 2.533 & $-4^{\circ} 54^{\prime}$ \\
\hline 30 & 2.187 & $-8^{\circ} 11^{\prime}$ & 2.073 & $-8^{\circ} 34^{\prime}$ \\
\hline 40 & 1.528 & $-15^{\circ} 7^{\prime}$ & 1.470 & $-14^{\circ} 50^{\prime}$ \\
\hline 50 & 0.748 & $-34^{\circ} 48^{\prime}$ & 0.891 & $-31^{\circ} 12^{\prime}$ \\
\hline 60 & -0.416 & $-115^{\circ} 58^{\prime}$ & -0.380 & $-97^{\circ} 10^{\prime}$ \\
\hline 70 & -0.793 & $-160^{\circ} 15^{\prime}$ & -0.749 & $-132^{\circ} 15^{\prime}$ \\
\hline 80 & -1.062 & $-172^{\circ} 33^{\prime}$ & -1.140 & $-171^{\circ} 54^{\prime}$ \\
\hline 90 & -1.148 & $-180^{\circ} 0^{\prime}$ & -1.284 & $-180^{\circ} 0^{\prime}$ \\
\hline & & (01̈1) plane & & \\
\hline 0 & 2.814 & $0^{\circ} 0^{\prime}$ & 2.910 & $0^{\circ} 0^{\prime}$ \\
\hline 10 & 2.716 & $1^{\circ} 29^{\prime}$ & 2.740 & $0^{\circ} 29^{\prime}$ \\
\hline 20 & 2.345 & $1^{\circ} 0^{\prime}$ & 2.260 & $0^{\circ} 27^{\prime}$ \\
\hline 30 & 1.643 & $-2^{\circ} 48^{\prime}$ & 1.566 & $-2^{\circ} 15^{\prime}$ \\
\hline 40 & 0.795 & $-16^{\circ} 50^{\prime}$ & 0.797 & $-15^{\circ} 17^{\prime}$ \\
\hline 50 & 0.327 & $-98^{\circ} 42^{\prime}$ & 0.319 & $-43^{\circ} 23^{\prime}$ \\
\hline 60 & -0.735 & $-153^{\circ} 37^{\prime}$ & -0.674 & $-156^{\circ} 40^{\prime}$ \\
\hline 70 & -1.088 & $-166^{\circ} 20^{\prime}$ & -1.082 & $-165^{\circ} 11^{\prime}$ \\
\hline 80 & -1.285 & $-173^{\circ} 45^{\prime}$ & -1.321 & $-173^{\circ} 13^{\prime}$ \\
\hline 90 & -1.348 & $-180^{\circ} 0^{\prime}$ & -1.424 & $-180^{\circ} 0^{\prime}$ \\
\hline
\end{tabular}

TABLE V. Comparison of actual and asymptotic lattice-statics results for a vacancy in $\mathrm{Al}$.

\begin{tabular}{ccc}
\hline \hline & $\begin{array}{c}\text { Lattice statics } \\
(\text { exact) } \\
|\xi| r^{2} \\
\text { Neighbor }\end{array}$ & $\begin{array}{c}\text { Asymptotic method } \\
|\xi| r^{2} \\
\left.\text { (units of } 2 a^{3}\right)\end{array}$ \\
\hline 110 & -0.0215 & -0.0307 \\
200 & -0.0605 & -0.0210 \\
211 & -0.0150 & -0.0304 \\
220 & -0.0247 & -0.0307 \\
222 & -0.0097 & -0.0343 \\
310 & -0.0360 & -0.0241 \\
330 & -0.0270 & -0.0307 \\
400 & -0.0278 & -0.0210 \\
411 & -0.0275 & -0.0253 \\
420 & -0.0325 & -0.0268 \\
422 & -0.0243 & -0.0304 \\
433 & -0.0309 & -0.0339 \\
440 & -0.0252 & -0.0307 \\
444 & -0.0323 & -0.0343 \\
\hline
\end{tabular}

where the interatomic forces we have used ${ }^{7}$ are of significantly long range, one would intuitively expect that the necessity to proceed to very large distances from the defect before one obtains a match between the lattice statics and continuum displacement fields will be even greater. To examine whether or not this is the case, we have carried out a full lattice-statics calculation for $\mathrm{K}$ out to the 40th neighbors along the three symmetry directions $\langle 100\rangle,\langle 110\rangle$, and $\langle 111\rangle$.

In the cases of the first two directions, wave-vector samples corresponding to a supercell containing 64000 lattice cells was used and in Figs. 8 and 9, we show plots of $|\xi| r^{2}$ versus $r$ for these two directions. In neither case does this quantity settle down to the asymptotic limit. The reason for this is that the displacements we are computing are obviously those appropriate to a superlattice of defects. Thus, when sufficiently far from a

TABLE VI. Comparison of exact and asymptotic lattice-statics results for an interstitial $\mathrm{Cu}$ atom in $\mathrm{Cu}$.

\begin{tabular}{|c|c|c|}
\hline Neighbor & $\begin{array}{l}\text { Lattice statics } \\
\qquad \text { (exact) } \\
|\mathfrak{\xi}| r^{2} \\
\left.\text { (units of } 2 a^{3}\right)\end{array}$ & $\begin{array}{l}\text { Asymptotic method } \\
|\xi| r^{2} \\
\text { (units of } 2 a^{3} \text { ) }\end{array}$ \\
\hline 100 & 0.0856 & -0.00977 \\
\hline 111 & -0.00591 & 0.1581 \\
\hline 210 & 0.09513 & 0.0312 \\
\hline 221 & 0.0507 & 0.1250 \\
\hline 300 & -0.00352 & -0.00977 \\
\hline 311 & 0.0722 & 0.0268 \\
\hline 320 & 0.0962 & 0.0753 \\
\hline 331 & 0.0784 & 0.1126 \\
\hline 333 & 0.1007 & 0.1581 \\
\hline 410 & 0.0133 & 0.00714 \\
\hline 430 & 0.0913 & 0.0803 \\
\hline 441 & 0.0907 & 0.1080 \\
\hline 443 & 0.1121 & 0.1471 \\
\hline 520 & 0.0277 & 0.0172 \\
\hline 522 & 0.0652 & 0.0436 \\
\hline 533 & 0.0992 & 0.1033 \\
\hline 540 & 0.0897 & 0.0835 \\
\hline 544 & 0.1204 & 0.1464 \\
\hline 630 & 0.0401 & 0.0312 \\
\hline
\end{tabular}




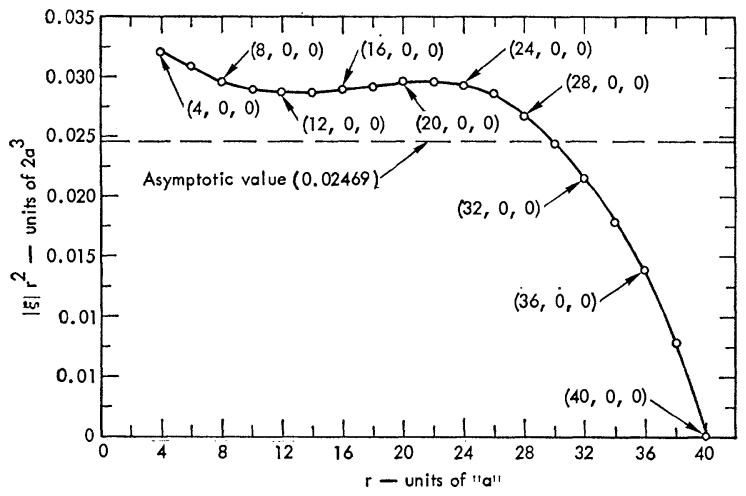

FIG. 8. $|\xi| r^{2}$ calculated from exact lattice statics, as a function of distance $r$ from the defect along $\langle 100\rangle$ in $K$. A sample of 64000 wave vectors was used. The dotted line shows the elastic limit predicted by the asymptotic theory.

given defect, the influence of the other defects in the superlattice becomes important. In these instances, we believe it is dominant beyond the 26th neighbors. However, our results do demonstrate that the displacement field about one defect has certainly not reached the asymptotic limit as far out as $(22,0,0)$ along $\langle 100\rangle$ and $(20,20,0)$ along $\langle 110\rangle$.

For the displacements along the $\langle 111\rangle$ directions we have made calculations for a sample of wave vectors corresponding to 512000 lattice cells (see Fig. 10). By judicious comparison of these results with those for a supercell containing 64000 lattice cells, we are able to estimate that the asymptotic regime is obtained at about the 27 th neighbor. The reason we are able to reach such a definitive conclusion for this class of directions is that the displacement field is particularly strong along the $\langle 111\rangle$ directions; thus the displacements produced by a given vacancy are not masked by those produced by the remaining defects in the superlattice before the asymptotic region is attained. These results enable us to assert categorically that, to obtain a proper representation of the displacement fields about vacancies in the alkali metals, it is necessary that any theoretical calculation treat a very large number of atoms on a discrete basis. It seems to us that lattice statics offers the only acceptable way of doing this.

An alternative method of calculating asymptotic displacements about a defect has recently been presented by Lie and Koehler. ${ }^{8}$ They start by using Fredholm's solution for the equations of elasticity in the absence of body forces. This reduces these sixth-order differential equations for the displacement components to sixthdegree polynomials. Using these polynomial solutions, they arrive at a Green's-function matrix $\mathbf{G}$. The element $G_{i j}$ of this matrix determines the displacement of a point in the $x_{i}$ direction due to a unit force at the origin in the $x_{j}$ direction. The solution of the equilibrium equations with a body force of density $F\left(r^{\prime}\right)$ is then

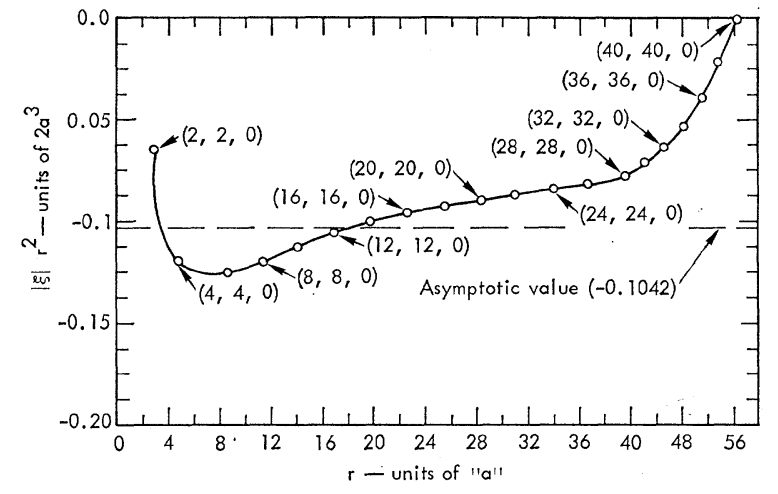

FIG. 9. $|\xi| r^{2}$ calculated from exact lattice statics, as a function of distance $r$ from the defect along $\langle 110\rangle$ in K. A sample of 64000 wave vectors was used. The dotted line shows the elastic limit predicted by the asymptotic theory.

given by

$$
\mathfrak{u}(\mathbf{r})=\iiint \mathbf{G}\left(\mathbf{r}-\mathbf{r}^{\prime}\right) \cdot \mathbf{F}\left(\mathbf{r}^{\prime}\right) d^{3} \mathbf{r}^{\prime}
$$

The elements of the Green's-function matrix $G_{i j}$ must be evaluated for each material considered. This involves determining the roots of the sixth-order polynomial equations, the coefficients of which are functions of the elastic constants and the polar and azimuthal angles $\theta$ and $\phi$ measured with respect to axes having the defect as their origin. It can then be shown that $|\mathbf{r}| G_{i j}$ is a function of $\theta$ and $\phi$ only. This function can be expressed as a double Fourier series in $\theta$ and $\phi$, the form of the series being determined by symmetry. Values of $|\mathbf{r}| G_{i j}$ can then be computed numerically for $\mathrm{Al}, \mathrm{Cu}$, and $\mathrm{Li}$ over a range of $\theta$ for several values of $\phi$. Using this approach, these authors calculated stresses which they estimated were accurate to about $15 \%$ for $\mathrm{Cu}$ and $8 \%$ for $\mathrm{Al}$. Stresses were not calculated for $\mathrm{Li}$ which has so high an

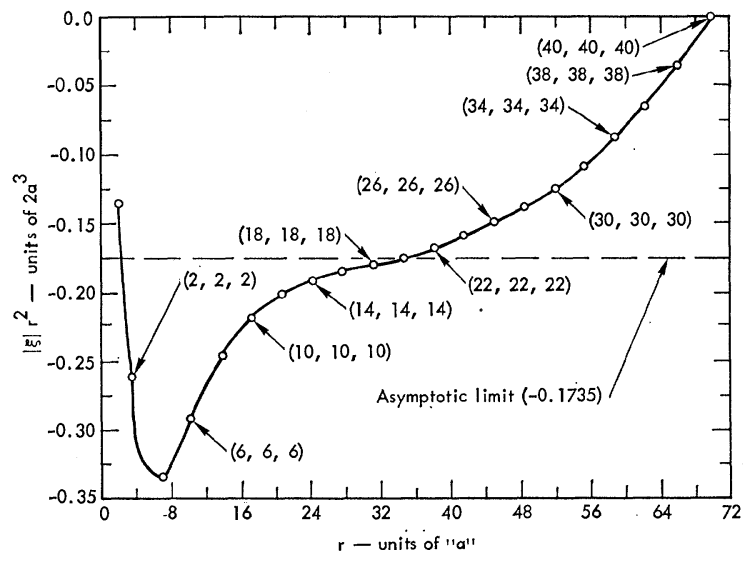

FIG. 10. $|\xi| r^{2}$ calculated from exact lattice statics as a function of distance $r$ from the defect along $\langle 111\rangle$ in $\mathrm{K}$. A sample of 512000 wave vectors was used. The dotted line shows the elastic limit predicted by the asymptotic theory. 
anisotropy that the Fourier series would have to contain a large number of terms to adequately represent the stresses. Fifteen terms were necessary in the stress series for $\mathrm{Cu}$ and six terms for $\mathrm{Al}$.

The displacements which we have computed using the series of Lie and Koehler are compared with our own results in Tables I-IV for various defect types. ${ }^{10}$ As can be seen from Table II the comparison between the two sets of results is excellent-always well within the $8 \%$ accuracy quoted by Lie and Koehler. The results for the double force in $\mathrm{Al}$, shown in Table IV also agree with each other but vary in some instances by as much as $11 \%$.

In the case of the $\mathrm{Cu}$ interstitial in $\mathrm{Cu}$, the difference between the two sets of results varies from about $50 \%$ in the [100] direction to about $10 \%$ in the [110] direction. The displacements along [100] are relatively small, however, so that one would expect that more terms in the series of Lie and Koehler would be necessary to obtain accurate values along this direction. The single double-force results for $\mathrm{Cu}$, shown in Table III are quite close to each other, both in magnitude and in angular deviation from the radial. As might be expected, the greatest differences in both magnitude and angle occur where the distortion of the radius $a$ sphere changes from an outward to an inward direction.

It should be pointed out that for the spherical defect problems, the Fourier series of Lie and Koehler do not give the same values at $(1,1,0)$ as at $(0,1,1)$. (These points are given, respectively, by $\epsilon=45^{\circ}$ and by $\epsilon=90^{\circ}$ in Tables I and II.) This discrepancy arises because the Fourier series for these two points involve different angles, and hence, different coefficients. Since the coefficients are obtained by curve fitting along different crystal directions, it cannot be expected that the same number of terms in the series for two equivalent points will give identical results. A second disadvantage to the curve-fitting techniques used by Lie and Koehler is that for complex defects, sharp details of the distortions are likely to be lost.

The asymptotic displacement values obtained from the method of lattice statics are exact in the elastic limit. The calculations are general, in that the elastic constants or force constants for a material can be in-

${ }^{10}$ Lie and Koehler represent their cubic defect as three mutually perpendicular double forces at the origin having unit elastic strength, i.e., $G=1$. Our cubic-defect models can also be represented as triple double-forces at the origin, but with forces adjusted to give the defect strengths shown in Figs. 1 and 2, derived for $\mathrm{Cu}$ and $\mathrm{Al}$ from exact lattice-statics calculations. Hence, in order to compare our results with those of Lie and Koehler we have multiplied their $G=1$ results by our own elastic strengths. serted directly into the program without intermediate curve fitting which gives coefficients for only one material. In addition the method of lattice statics is independent of the anisotropy of the material used and is a natural extension of atomistic theory to continuum elasticity-a transition which is not contained in the method of Lie and Koehler.

The curves showing displacements per unit force about spherical defects in $\mathrm{Li}$ are shown in Figs. 5(a) and 5(b). These results cannot be compared with numerical values calculated using exact lattice statics or with the alternative asymptotic method of Lie and Koehler. It is apparent, however, that the distortion in $\mathrm{Li}$, follows much the same pattern as it does in the other alkali metals.

\section{SUMMARY}

We have applied the method of lattice statics to the calculation of asymptotic displacements at large distances about various classes of defects in $\mathrm{Al}, \mathrm{Cu}, \mathrm{Na}$, $\mathrm{K}$, and Li. We have also calculated the distortions due to unit single double-forces along [100] in $\mathrm{Al}$ and $\mathrm{Cu}$. It appears to us that the only consistent method for calculating the direct-space forces due to the defect and arriving at an accurate strength parameter $G$ is to perform these calculations using lattice statics and the prescription for $G$ given by Hardy. ${ }^{2}$ Once this is done, the asymptotic distortions can be found by the method presented in this paper.

The distortions can also be calculated using the method due to Lie and Koehler if the crystal is reasonably isotropic. However, for many materials of interest, this method does not give accurate results unless a large number of terms are included in their Green'sfunction expansions. In addition, the curve-fitting techniques used to arrive at the coefficients of the Green'sfunction expansions are likely to obscure fine details of the distortion profiles.

The most important result of the present work is that, in each case where the exact results could be compared with the results of the asymptotic theory, the former did not settle down to the elastic limit until one was considering atoms that were many interatomic spacings from the defect.

It thus seems that the approach we have described in this paper, which is a logical extension of the method of lattice statics for the computation of displacements in the vicinity of the defect to the computation of longrange displacements, offers the most precise and flexible way of solving this problem. 